\title{
Correction to: The recurrent missense mutation p.(Arg367Trp) in YARS1 causes a distinct neurodevelopmental phenotype
}

\author{
Luisa Averdunk ${ }^{1,2} \cdot$ Heinrich Sticht ${ }^{3} \cdot$ Harald Surowy ${ }^{1} \cdot$ Hermann-Josef Lüdecke ${ }^{1} \cdot$ Margarete Koch-Hogrebe ${ }^{4}$. \\ Hessa S. Alsaif ${ }^{5} \cdot$ Kimia Kahrizi $^{6} \cdot$ Hamad Alzaidan $^{5} \cdot$ Bashayer S. Alawam $^{5} \cdot$ Mohamed Tohary $^{5} \cdot$ Cornelia Kraus $^{7}$. \\ Sabine Endele ${ }^{7} \cdot$ Erin Wadman $^{8} \cdot$ Julie D. Kaplan $^{8} \cdot$ Stephanie Efthymiou $^{9} \cdot$ Hossein Najmabadi $^{5} \cdot$ André Reis $^{7}$. \\ Fowzan S. Alkuraya ${ }^{5}$. Dagmar Wieczorek ${ }^{10}$
}

Published online: 18 October 2021

(c) Springer-Verlag GmbH Germany, part of Springer Nature 2021

Correction to: Journal of Molecular Medicine https://doi.org/10.1007/s00109-021-02124-9

In the published original paper, Fig. 1 is incomplete. Only Fig. 1a is shown. This erratum corrects Fig. 1 and adds the missing panel $1 \mathrm{~b}$-f to the article.

The original article can be found online at https://doi.org/10.1007/ s00109-021-02124-9.

Dagmar Wieczorek

dagmar.wieczorek@hhu.de

1 Institute of Human Genetics, Medical Faculty and University Hospital Düsseldorf, Heinrich-Heine-University Düsseldorf, Universitätsstraße 1, 40225 Düsseldorf, Germany

2 Department of General Pediatrics, Neonatology and Pediatric Cardiology, Medical Faculty and University Hospital Düsseldorf, Heinrich-Heine-University, Düsseldorf, Germany

3 Division of Bioinformatics, Institute of Biochemistry, Friedrich-Alexander-Universität Erlangen-Nürnberg, Erlangen, Germany

4 Children's Hospital Datteln, University Witten Herdecke, Datteln, Germany

\section{References}

1. UniProt C (2019) UniProt: a worldwide hub of protein knowledge. Nucleic Acids Res 47(D1):D506-D515. https://doi.org/10.1093/ nar/gky1049

Publisher's Note Springer Nature remains neutral with regard to jurisdictional claims in published maps and institutional affiliations.
5 Department of Genetics, King Faisal Specialist Hospital and Research Center, Riyadh, Saudi Arabia

6 Genetics Research Center, University of Social Welfare and Rehabilitation Sciences, Tehran, Iran

7 Institute of Human Genetics, University Hospital Erlangen, Friedrich-Alexander-Universität Erlangen-Nürnberg, Erlangen, Germany

8 Division of Medical Genetics, Department of Pediatrics, DuPont Hospital for Children, Nemours Alfred I, Wilmington, Delaware, DE, USA

9 Department of Neuromuscular Diseases, UCL Queen Square Institute of Neurology, The National Hospital for Neurology and Neurosurgery, London, UK 
a

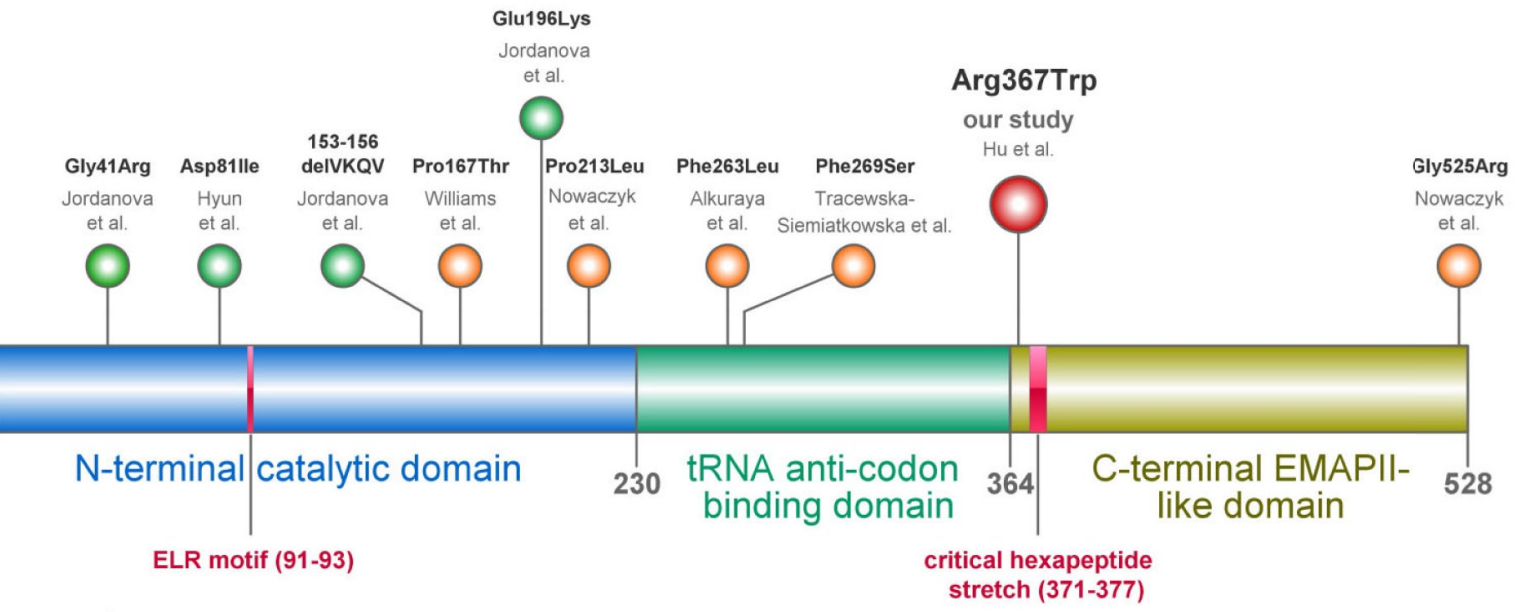

b

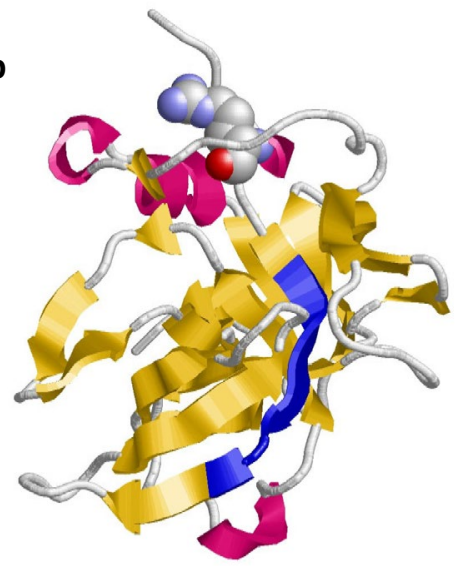

e

Homo sapiens

Danio rerio $\quad 361$

Xenopus tropicalis 360

Mus musculus 360

Bos Taurus $\quad 360$

Rattus norvegicus 360

Pongo abelli $\quad 360$

Gallus gallus $\quad 359$
C

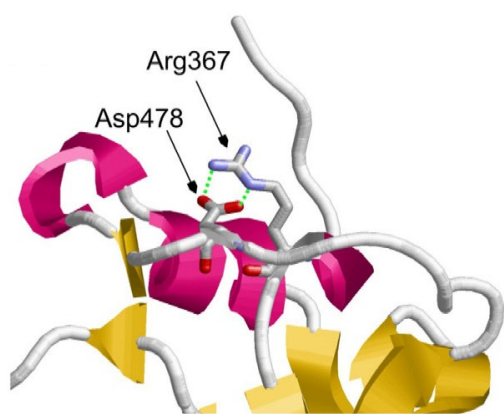

PEEVIPSRTDIRVGKIIMVEHPDADSIYVEKIDVGEAEPRTVVGLVEVPKEELQDRL DDEVIPGR DIRVGKVISVERHPDADSLYLERIDVGEEQPRTVVSGLVAYITEEQLQDRI PEEIDPSIDLRVGKILSVROHPDADSUYVESVDVGEENPRCVVSGLVYVPSDQLIGRS PEEVIPSR DIRVGKILSVERHPDADSIYVERIDVGEAEPRTVSGLVQVVREELQDRI PEEVIFSRDIRVGKVISVDKHPDADSLYVERIDVGEAEPRTVVSGLVEVPKEELQDRI PEEIIPSRDIRVGKILSVEKHPDADSLYVERIDVGEAEPRTVVSGLVEVPKEELQDRI PEEVIPSR DIRVGKITVEKHPDADSIYVEKIDVGEAERRIVVSGLVQEVPKEELQDRI PETIVP SE DIRVGKVVSVERHPDADSIYVERIDVGEPEPRTVVSGUVEVPREQIQDRT

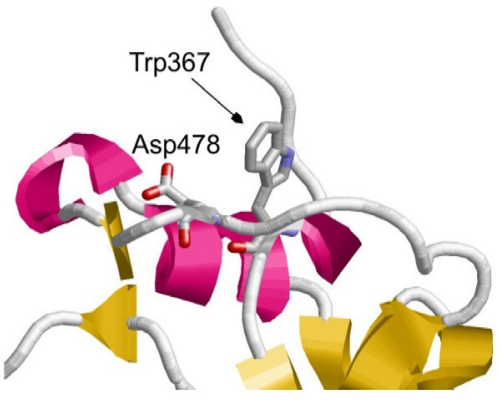

f
Fig. 1 (a) Organization of the three domains of human TyrRS and biallelic variants reported in the literature (orange dots). Three domains: (i) The catalytic N-terminal domain is essential for aminoacylation of tRNA. As a monomeric fragment, the N-terminal domain has cytokine activity and the ELR motif is critical for this activity. (ii) tRNA anti-codon binding domain (iii) The C-terminal EMAP-II-like domain that was shown to be dispensable for aminoacylation. The heptapeptide sequence within this domain is critical for the cytokine activity. The homozygous variants p.(Pro167Thr) and p.(Pro213Leu) reside in the catalytic domain, harboring to the critical ELR motif, while most of the other variants reside outside this domain (orange dots). All five heterozygous mutations causing Charcot-Marie-Tooth neuropathy reside in the catalytic domain (green dots). (b) Structure of the C-terminal domain of TyrRS shown as backbone representation indicating the elements of secondary structure. Arg367 is shown in space-filled presentation and colored according to the atom types. The heptapeptide sequence stretch critical for the cytokine activity (residues 371-377) is shown in blue. (c) Structural role of Arg367 in TyrRS: In the wildtype, Arg367 forms a salt-bridge to Asp478 (indicated by green dotted lines). (d) In the p.(Arg367Trp) mutant, the bulky uncharged tryptophan cannot form an electrostatic interaction resulting in domain destabilization. $(\mathbf{c}+\mathbf{d})$ Arg367/Asp478 are shown in stick presentation. (e) Multi-species amino acid alignment indicates that Arg367 is highly conserved in mammals and down to zebrafish (danio rerio), but not in the western clawed frog (xenopus tropicalis) [1] (f) Amino acid alignment of the C-terminal EMAP-II-like domain of TyrRS compared to AIMP1. The corresponding residues Arg367 and Asp478 are conserved in both domains. TyrRS, tyrosine tRNA synthetase. AIMP1, Aminoacyl tRNA Synthetase Complex Interacting Multifunctional Protein 1 